\title{
BRIGADA DE PRIMEROS AUXILIOS COMO ESTRATEGIA PARA LA ENSEÑANZA \\ DE MEDIDAS DE PREVENCIÓN DE LA SALUD ESCOLAR

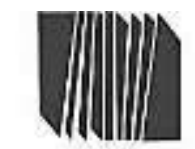

UNEVUKABDAD PLDAEDOICA EDTH MECATAL LIECHTAD O

\section{Revista}

Órgano Divulgativo de la Subdirección de Investigación y Postgrado del Instituto Pedagógico de Barquisimeto "Luis Beltrán Prieto Figueroa"

Barquisimeto Estado Lara

\begin{tabular}{|c|}
\hline Enero - Abril 2018 \\
\hline $\begin{array}{l}\text { BRIGADA DE PRIMEROS AUXILIOS COMO ESTRATEGIA PARA LA ENSEÑANZA } \\
\text { DE MEDIDAS DE PREVENCIÓN DE LA SALUD ESCOLAR }\end{array}$ \\
\hline $\begin{array}{c}\text { FIRST AIDS BRIGADE AS STRATEGY FOR THE TEACHING OF HEALTH } \\
\text { PREVENTIVE MEASURES AT SCHOOLS }\end{array}$ \\
\hline
\end{tabular}

AUTOR

Maritza Álvarez (MPPPE)

Yolanda Montoya (UPEL) 


\section{BRIGADA DE PRIMEROS AUXILIOS COMO ESTRATEGIA PARA LA ENSEÑANZA \\ DE MEDIDAS DE PREVENCIÓN DE LA SALUD ESCOLAR \\ Maritza Álvarez y Yolanda Montoya}

(pp 57-82)

\section{Investigación}

Recibido: 03-07-2017

\section{RESUMEN}

En las organizaciones de Venezuela suele observarse la ausencia de comisiones de estudiantes y docentes dispuestos a actuar con los primeros auxilios básicos, en atención inmediata ante un evento de enfermedad o lesión física de alguna persona del ambiente educativo. De acuerdo al planteamiento anterior, esta investigación tiene el objetivo de conformar una brigada escolar de primeros auxilios básicos como estrategia para la enseñanza de medidas de prevención en salud. El diseño en una investigación cualitativa de acción participante bajo un paradigma socio-crítico. La técnica usada fue la entrevista semiestructurada aplicadas a los informantes claves: cinco (5) estudiantes. Los hallazgos constataron la necesidad de planificar y ejecutar el plan de acción de "brigadas de primeros auxilios básicos y prevención de la salud. Se concluye con la reflexión final: la organización de los grupos, aportan actividades de primeros auxilios y de prevención de riesgo en la escuela, favoreciendo a una conciencia voluntaria y social para servir ante evento adverso.

Descriptores:brigada de primeros auxilios, enseñanza de salud, prevención

\author{
Maritza Álvarez (MPPPE) ${ }^{*}$ \\ Yolanda Montoya (UPEL-UNEFA) ${ }^{* *}$
}

Aceptado: 22-01-2018

\begin{abstract}
In Venezuelan organizations it is used to observe the lack of students and teachers commissions that are able to use basic first aids for immediate attention in the case of any person' sickness or physical lesion at schools. So that, this study is aimed to make up a basic first aids school brigade as strategy for the teaching of health preventive measures. It is an action participant research, on the basis of the qualitative approach and social-critical paradigm. The semi-structured interview technique was used, which was applied to five (5) students who acted as key informers. The findings lead to evidence the necessity of planning and applying an action plan to make up basic first aids and health prevention brigades. As a final reflection, it is considered that these kinds of groups benefit schools because people develop voluntary and social conscience to serve in possible dangerous situations.
\end{abstract}

Key words: first aids and health prevention brigades

\footnotetext{
* Profesora de E.B.E “General Diego Ibarra”. Profesora. Especialista en Procesos Didácticos en la Educación Básica. maritzaalvarez4@gmail.com

${ }^{* *}$ Profesora Contratada de Universidad Pedagógica Experimental Libertador (IMPM-UPEL). y Universidad Nacional Experimental Politécnica de La Fuerza Armada Nacional (UNEFA) Carabobo. Lcda. en Educación. Magister en Educación. Doctor of Management in Educational Institución yolandamontoya@ hotmail.es
} 


\section{INTRODUCCIÒN}

La educación es el término vinculante con el progreso de la calidad de vida de los individuos y las comunidades. Favoreciendo la actualización, información y actividades que desarrollen sus capacidades, a su vez comprometidos en la identificación y control de los recursos acordes para promover el bienestar, al mismo tiempo de brindar los conocimientos y alternativas necesarias para la prevención de hechos en el desarrollo personal y social.

Es de sumo interés organizar proyectos de prevención de accidentes en el espacio educativo, considerando el suministro básico de insumos para prestar los primeros auxilios al momento de presentarse emergencias en las instituciones educativas, y en todos espacios acreedores de implementación y organización de Brigadas Escolares que apoyen la capacitación y actuación ante una eventualidad, emergencia o siniestro.

En consecuencia, en la actualidad este tema es relevante debido a que el estudiante pasa una importante parte del día en la escuela, llamados a conformar una comisión o brigada preparada al servicio del bienestar estudiantil en el área de salud dentro de la corporación educativa. De allí, el trabajar con niños y niñas no exime a las escuelas de que se registren pequeños accidentes, los cuales, ocurren de manera imprevista, considerándose como momentos más vulnerables y de mayor riesgo la hora del recreo, las actividades desplegadas en Educación Física y dentro del salón de clases.

Evidentemente, un accidente escolar es un evento no previsto y cuya responsabilidad, en este caso los docentes, es evitar en la medida de las posibilidades la ocurrencia de los accidentes, ya que de una u otra forma ellos son los que permanecen en contacto directo con los estudiantes durante el desempeño de la labor pedagógica. No obstante, la brigada de primeros auxilios básicos como estrategia para la atención primaria de eventos asociados a la salud, constituye una respuesta a una necesidad latente en la sociedad actual y asimismo en el ámbito escolar. 
En tal sentido, dentro del marco educativo, los accidentes escolares representan un considerable problema de salud. Además de los riesgos y amenazas presentes en las organizaciones escolares y los autores involucrados de la sociedad. De allí, la importancia de los Primeros Auxilio, con clarificada interpretación en su definición, según el Manual de Emergencias Médicas Fundación Atención Inmediata (1995): "Es la atención o cuidado que se le proporciona a una persona víctima de un accidente o enfermedad repentina hasta tanto se pueda contar con los servicios médicos o se pueda trasladar a un centro asistencial más cercano"(p.19).

Sobre la base de los argumentos anteriores, uno de los aspectos importantes en la sociedad de este siglo es la culturización de la población para estar atenta y menos vulnerable ante los accidentes cotidianos de la escolaridad. En este escenario, la dinámica actual a nivel educativo, genera necesidades que son de gran importancia en el buen desarrollo y funcionamiento de las actividades dentro de una institución educativa, siendo una de las prioridades la protección al estudiante y de los demás miembros que conforman la institución. De acuerdo a las condiciones de seguridad, éstas deben tender siempre a su protección tanto física como mental, lo que permitirá un mejor rendimiento académico para beneficio del estudiante, y de la institución.

De acuerdo a lo planteado, los docentes en su mayoría demuestran interés en el aprendizaje de las diferentes áreas así como la integración de las las Tecnologías de la Información y la Comunicación (TICs), en el proceso de enseñanza, lo cual, va a permitir la adquisición de conocimiento de los Primeros Auxilios Básicos y que serán implementados de la brigada con el objetivo de prevención en lesiones en el cuerpo humano, accidentes tanto en el hogar como en desastres naturales, donde el docente juega un papel primordial en el uso de estrategias innovadoras. Es vital despertar conciencia sobre la importancia que representa el tener los conocimientos y la dotación básica de insumos de Primeros Auxilios en la escuela, tomando en cuenta que toda actividad preventiva tiene como base fundamental la educación. 
En Venezuela, para cumplir con las emergencias hay que llamar 171 Emergencia, C.A. Es una organización de "Centro de Atención”, el cual presta sus servicios en todo momento y su trascendencia es a todo el país. Cabe señalar que obra en conjunto con la dirección nacional de protección civil (PC), la cual atiende y ejecuta actividades de prevención a toda persona en situación de amenaza. Pues bien, las dos (2) organizaciones ayudan a la población del país a mantenerse a salvo o proteger a los individuos, en situaciones de desastre o emergencia. De la misma manera los organismos son capaces de proceder al amparo del ciudadano, pero éstos de algún modo requieren ayuda para la preparación individual de la población, y así los habitantes estén preparados en los primeros auxilios como: conocimientos, maniobras y técnicas referentes al tema, los organismos obtendrían una mayor tiempo de respuesta y mayor entorpecimientos al llegar a la situación de riesgos.

En este sentido, la problemática del $5^{\circ}$ grado sección " $D$ ” Escuela Básica Estadal “General Diego Ibarra”, Parroquia Miguel Peña del Municipio Valencia Estado Carabobo. Venezuela, a través de la observación participante, se observó que durante el recreo los niños juegan, corren, se empujan y hasta en la clase de educación Física, en ocasiones, se han registrado niños lesionados, hasta desmayos sin tener a la mano los primeros auxilios. Considerándose, accidentes básicos que generan tensión y preocupación ante estos eventos por los docentes y discentes, además de la falta de preparación del personal y estudiantes ante dichas situaciones de emergencia escolar.

Por consiguiente, es importante para este estudio, instruir a los estudiantes en la aplicación de estrategias de primeros auxilios en educación para la salud y la dotación de insumos para lesiones o emergencia leves, que puedan ser atendidas en la misma institución. Por ende, es vital despertar conciencia sobre la importancia que representa el tener los conocimientos y la dotación básica de insumos de Primeros Auxilios básicos en la escuela, tomando en cuenta que toda actividad preventiva tiene como base fundamental la educación. Cuyo propósito en este estudio es: 


\section{BRIGADA DE PRIMEROS AUXILIOS COMO ESTRATEGIA PARA LA ENSEÑANZA \\ DE MEDIDAS DE PREVENCIÓN DE LA SALUD ESCOLAR \\ Maritza Álvarez y Yolanda Montoya \\ (pp 57-82)}

Conformar una brigada de primeros auxilios como estrategia para la enseñanza de medidas de prevención de la salud escolar de los estudiantes de la Escuela Básica Estadal "General Diego Ibarra" ubicada en la parroquia Miguel Peña del municipio Valencia, estado Carabobo.

Diagnosticar los conocimientos previos de primeros auxilios que tienen los y las estudiantes de la Escuela Básica Estadal "General Diego Ibarra" ubicada en la parroquia Miguel Peña del municipio Valencia, estado Carabobo.

Planificar actividades para la conformación de la brigada escolar como estrategia de prevención y atención de salud escolar de la Escuela Básica Estadal "General Diego Ibarra"

\section{ARGUMENTACIÓN TEÓRICA}

\section{Primeros Auxilios}

De acuerdo con los planteamientos anteriores, es oportuno abordar lo referente a los primeros auxilios, centro de esta investigación. Según, Castro (2006) indica que "los Primeros Auxilios son los cuidados inmediatos, adecuados y temporales prestados a las personas accidentadas o con enfermedad, antes de ser atendidos en un centro asistencial"'(p.15), lo cual exige en la actualidad, afrontar los aspectos físicos o biológicos, y así brindar primeros auxilios de forma adecuada aun sin poseer conocimientos y habilidades universitarias. En pocas palabras, no es indispensable poseer una licenciatura en psicología para estar en condiciones de reconfortar a una persona que ha sufrido un accidente, Fabris(s/f) dice:

Los primeros auxilios son las medidas terapéuticas urgentes que se aplican a las víctimas de accidentes o enfermedades, repentinas hasta disponer de tratamiento especializado. El propósito de los primeros auxilios es aliviar el dolor y la ansiedad del herido o enfermo y evitar el agravamiento de su estado. En casos extremos son necesarios para evitar la muerte hasta que se consiga asistencia médica (p.28) 
A su vez, considerando la magnitud y la diversidad de problemas que tiene relación con la accidentalidad escolar, ningún sector aislado puede responsabilizarse por sí solo de esos problemas. Cada escuela u organización educativa debe establecer e implementar normas precisas de acción que garanticen a los estudiantes la gestión en un ambiente escolar con posibles condiciones inseguras y una apropiada instrucción de las reglas para conservar la salud y evitar accidentes.

De este modo, los derechos y deberes del colectivo estudiantil pueden disfrutar de un ambiente sano y ecológicamente equilibrado con atención a la integridad física, psíquica y moral, entre otras. [Art. 8 y 93 de la Ley Orgánica para la Protección de Niños, Niñas y Adolescentes (LOPNNA)]. Por lo anterior es necesario que los niños y niñas, tengan conocimiento de los principios, normas y técnicas de primeros auxilios, ya que hay situaciones y circunstancias que rigen a actuar rápida y oportunamente en pro de salvar una vida: ya sea la de un desconocido, la de un familiar, la de un compañero o inclusive la propia.

Según la Cruz Roja de Venezuela (2006); la meta es "Un socorrista por cada familia”, ya que considera que los primeros auxilios, más que limpiar una herida, es saber hacerlo acompañado de un gesto humanitario que permita atender de una manera integral al individuo. La Federación Internacional de Sociedades de la Cruz Roja y de la Media Luna Roja, desempeña un papel de liderazgo en la prestación de primeros auxilios y en la formación de esa labor, donde se adopta un enfoque holístico de los Primeros Auxilios, que contempla las necesidades físicas, sociales y psicológicas de los heridos, de sus familias y de los propios voluntarios. Asimismo, para la Cruz Roja (ob.cit.)

La preparación es un componente clave de todos los programas de Primeros Auxilios de la Cruz Roja, los servicios y los cursos de formación en esta materia se utilizan como puertas de entrada para los proyectos de preparación para desastres y educación comunitaria”. La Cruz Roja Venezolana, es reconocida como una escuela formadora de Primeros Auxilios en nuestro país e incentiva a la ciudadanía sobre la importancia en el área de los primeros auxilios.(s/n) 
Como indica la cita anterior, los primeros auxilios son contenidos de la Cruz Roja Venezolana, ente encargado de instruir a los ciudadanos sobre la prevención, reacción y emergencia ante eventos de desastres naturales o socioculturales, o riesgos físicos.

\section{Brigadas Escolares de Primeros Auxilios}

Son grupos organizados de jóvenes estudiantes, partícipes de acciones determinadas en cada comunidad educativa, son asesoradas y guiadas por un docente a fin de prevenir algún riesgo. Dentro de esta perspectiva, el estudio se desarrolla dentro de la escuela, estos riesgos se toman como físicos como los que pueden ocasionar los mismos estudiantes como empujones, caídas, juegos con útiles que pueden llegar a lastimar a otros. Lo que se persigue es lograr que exista una cultura de la prevención en función de los estudiantes, para tal efecto, la capacitación se realiza con el objetivo de culturizar a la población estudiantil. Además de gestionar que los brigadistas cuenten con características de: Franca disposición de colaboración, capacidad de organización y liderazgo, aceptación de sus compañeros y responsabilidad e iniciativa (Gabari y Buitrago, 2006).

\section{Brigadas Escolares para fomentar la Salud}

Ciertamente la brigada es un pequeño número de estudiantes voluntarios, para acciones definidas con un propósito determinado. De manera que el trabajo se manejó y se desarrolló en forma preventiva con objetivos imponentes a reforzar los contenidos propuestos por la comunidad educativa.

Siguiendo este orden de ideas, la organización de una Brigada Escolar debe orientarla y monitorearla el docente y contar por lo general con el apoyo de algún especialista que dirige su formación. Ante este escenario, el funcionamiento de estas brigadas en las escuelas, no afecta el normal desarrollo de los programas escolares, por el contrario, ofrece a los docentes posibilidades específicas, en estimular a los niños y jóvenes el espíritu de iniciativa, el sentido de grupo, el sentido de organización, cooperación y el sentimiento de ayuda, con proyecciones hacia la comunidad educativa. 
En este sentido, cada brigada escolar tiene el acuerdo en líneas normales de dos docentes, como mínimo, que se desempeñan como coordinadores de éstas. Estos educadores se distinguen por su sensibilidad social, espíritu de servicio desinteresado, sacrificio, colaboración, capacidad, iniciativa, puntualidad, y sentido de responsabilidad.

A su vez, la brigada escolar se ocupa de incentivar a la población estudiantil en general, en participar en los diferentes movimientos de la institución, con el fin de crear un conocimiento de riesgo a mediano plazo y que los y las estudiantes sean entes multiplicadores de las bondades del servicio que se le presta a la población de manera gratuita. Está conformada por un grupo de jóvenes escolares que persiguen como fin común la cooperación, disciplina, organización y sentimiento de ayuda en pro de la comunidad escolar y/o comunidad en general. En lo que respecta a las brigadas escolares de primeros auxilios, cabe decir que son grupos organizados de niños, niñas y jóvenes estudiantes que colaboran en acciones determinadas en cada comunidad educativa y que son asesoradas por los docentes que orientan pedagógicamente los fines y objetivos de estas organizaciones. (Morrongiello \& Lasenby-Lessard, 2007).

Tal como se ha dicho, las brigadas escolares de salud tienen una amplia escala de funciones que van desde la propia preparación de los integrantes de la misma, así como también la elaboración y desarrollo de sesiones educativas y actividades destinadas a sus propios compañeros con el fin de transmitir un mensaje de prevención de enfermedades y protección de estilos de vida saludable, hasta una actuación de relevante importancia en situaciones de emergencia dentro de la institución educativa.

En atención a los razonamientos anteriores, el docente planifica el contenido académico de educación para la salud, deberá considerar el uso de los recursos, estrategias y técnicas para fomentar un ambiente abierto a la solución de problemas y al trabajo interactivo, que sólo será posible si se motiva a los estudiantes a la integración de los equipos de trabajo de las brigadas de primeros auxilios y así sean promotores de ideales, solidaridad, empatía y calidad humana en un aprendizaje significativo.(Little \& Wyver, 2010). 


\section{TEORÍAS QUE SUSTENTAN LA INVESTIGACIÓN}

\section{La Teoría del Aprendizaje de Lev Vygotsky}

La Zona de Desarrollo Próximo (ZDP) es central en la teoría socioconstructivista, sobre todo en los análisis que en base a ella se hacen sobre las prácticas educativas y en el diseño y desarrollo de estrategias escolares, por tanto se centran en ella las posturas de la línea pedagógica. Esta noción o concepto de ZDP articula o integra primero, para su propia comprensión el término de ZDR (Zona de Desarrollo Real) y, después las categorías de interacción social e internalización, así como la interrelación entre los procesos de aprendizaje y desarrollo.

Como preámbulo a la cita de Vygotsky sobre ZDP resulta interesante reflexionar sobre lo que De Lima (2000), la cual, dice sobre algunas palabras de Leóntiev, citadas a su vez por Wertsch: Los investigadores americanos se dedican constantemente a averiguar cómo el niño llega a ser lo que es; en la URSS se intenta descubrir no cómo el niño ha llegado a ser lo que es, sino cómo puede llegar a ser lo que no es”. Este señalamiento, continúa De Lima (ob,cit), relación de forma inmediata, con el conocimiento de Zona de Desarrollo Próximo de Vygotsky, que él la refiere: "no es otra cosa que la distancia entre el nivel real de desarrollo, determinado por la capacidad de resolver independientemente un problema, y el nivel de desarrollo potencial determinado a través de la resolución de un problema bajo la guía de un adulto o en colaboración con otro compañero más capaz".

Lo anterior permite elaborar una diferenciación entre los estudios de Piaget y Vygotsky, el primero, analiza el nivel del desarrollo real del niño o sea cómo el niño "ha llegado a ser lo que es" puesto que esta Zona de Desarrollo Real, refiere funciones que ya han madurado, es decir, los productos finales del desarrollo". En este nivel están los test y pruebas de diagnóstico para conocer el Coeficiente Intelectual (CI) y las preparaciones reales o previas de los sujetos.

En orden de ideas, Vygotsky (1998), al decir que la Zona de Desarrollo Próximo (ZDP), aborda aquellas funciones no maduradas, pero en proceso de maduración, esas funciones 
aproximante lograrán su madurez, se indicaría que él investigó "cómo el sujeto que aprende puede llegar a ser lo que no es”. Es aquí donde entra en función el papel del docente, del adulto, del compañero más capaz, en sí, la función de la interacción social para el logro del aprendizaje, de la internalización del conocimiento.

Lo anterior queda reforzado con esta cita del propio Vygotsky: "El nivel de desarrollo real caracteriza el desarrollo mental retrospectivamente, mientras que la zona de desarrollo próximo caracteriza el desarrollo mental prospectivamente", es decir, la ZDP potencia el conocimiento, el aprendizaje y en consecuencia el desarrollo o sea que el aprendizaje produce desarrollo y no el desarrollo propicia aprendizaje como lo propone Piaget.

En consecuencia, la zona de desarrollo próximo posibilita el desempeño antes que la conquista de una competencia. Cada alumno es portador de distintos niveles de desarrollo próximo posibles, en relación con las distintas áreas de desarrollo personal y respecto de las diferentes tareas y contenidos escolares. Desde este marco vigotskyano, los alumnos pueden ser considerados también recursos de mediación por parte del docente, por ejemplo cuando el estudiante más competente en una determinada tarea ayuda a otro compañero que necesita de su ayuda, constituye una situación por demás educativa

\section{Teoría de David Ausubel (1918)}

Por otro lado, Ausubel (1978) denomina que el conocimiento obtenido por el estudiante posea se estructura en forma cognitiva y se fusiona con el tema de estudio. pues así el aprendizaje se optimatiza. Otro factor importante son los preconceptos (conocimiento espontáneo de algo) ya que estos pueden determinar el éxito o fracaso en el aprendizaje, los preconceptos están arraigadas en la estructura cognitiva.

El individuo asimila mediante "Aprendizaje Significativo", se entiende por aprendizaje significativo a la incorporación de la nueva información a la estructura cognitiva del individuo. Esto creará una asimilación entre el conocimiento que el individuo posee en su estructura cognitiva con la nueva información, facilitando el aprendizaje.El conocimiento no se encuentra así por así en la estructura mental, para esto ha llevado un proceso ya que 
en la mente del hombre hay una red orgánica de ideas, conceptos, relaciones, informaciones, vinculadas entre sí y cuando llega una nueva información, ésta puede ser asimilada en la medida que se ajuste bien a la estructura conceptual preexistente, la cual, sin embargo, resultará modificada como resultado del proceso de asimilación.

\section{Características del aprendizaje significativo}

(a) Existe una interacción entre la nueva información con aquellos que se encuentran en la estructura cognitiva. (b) El aprendizaje nuevo adquiere significado cuando interactúa con la noción de la estructura cognitiva. (c) La nueva información contribuye a la estabilidad de la estructura conceptual preexistente.

\section{Tipos de aprendizaje significativo}

1.- El aprendizaje de representaciones que consiste en que el individuo atribuye significado a símbolos (verbales o escritos) mediante la asociación de éstos con sus referentes objetivos. Esta es la forma más elemental de aprendizaje y de ella van a depender los otros dos tipos.

2.- El aprendizaje de conceptos es, en cierto modo, siempre un aprendizaje de representaciones con la diferencia fundamental, por un lado, es la simple asociación símbolo - objeto, sino símbolo - atributos genéricos. Es decir, en este tipo de aprendizaje el sujeto disocia de la realidad objetiva de los atributos comunes a los objetos que les hace pertenecer a una cierta clase. El autor, define los "conceptos" como "objetos, acontecimientos, situaciones o propiedades que tienen atributos de criterios comunes y diseñados en cualquier cultura dada mediante algún símbolo o signo aceptado".

3.- En el aprendizajeno se trata de asimilar el significado de términos o símbolos aislados sino de ideas que resultan de una combinación lógica de términos en una sentencia. Por supuesto que no podrá tener lugar el aprendizaje de una proposición, a menos que los conceptos que en ella están incluidos, no hayan sido aprendidos previamente; de allí que lo 


\section{BRIGADA DE PRIMEROS AUXILIOS COMO ESTRATEGIA PARA LA ENSEÑANZA \\ DE MEDIDAS DE PREVENCIÓN DE LA SALUD ESCOLAR \\ Maritza Álvarez y Yolanda Montoya \\ (pp 57-82)}

aprendizajes de representaciones y de conceptos sean básicos para un aprendizaje de proposiciones.

En este sentido, las brigadas de primeros auxilios deben ser estimadas como un acto de encuentro de sus participantes a la elaboración conjunta de aprendizajes significativos, al crear una reflexión del contacto entre ellos, produciéndose así una diversidad de momentos generadores de emociones, interrogantes y reflexiones, los cuales, dependen de la interacción y las alcances elaboradas por los estudiantes, dado que son los impulsadores en el hacer y rehacer del conocimiento para las medidas de prevención ante diferentes riesgos en el contexto escolar.

\section{ASPECTOS METODOLÓGICOS}

\section{Paradigma Socio crítico}

Este estudio se apoya en el paradigma socio-crítico, en la crítica social con un marcado carácter auto-reflexivo. Contempla que el conocimiento se construye siempre por intereses de las necesidades de los grupos e intenta la autonomía racional y liberadora del ser humano. Por ello, este prototipo tiene el objetivo de formar personas que desarrollen su capacidad de reflexión crítica y les permita analizar su propio contexto y realidad cotidiana. (Sotelo, 2012. Sobre la base de este principio la presente investigación se apoya en la concepción holística y el paradigma socio-crítico, que según Carr y Kemmis, (1983), citado por La Madriz (2013):

La "investigación social crítica, incluida la acción social en la acción emancipatoria, concibe la educación como un proceso histórico e ideológico. Utiliza el razonamiento práctico por un interés emancipador de transformar las organizaciones y prácticas educativas para conseguir la racionalidad y justicia social". (p.188).

De allí, el contexto educativo, en el pospositivismo, permite el cuestionamiento auto reflexivo, llevada a cabo por los propios participantes en determinadas ocasiones con la finalidad de mejorar la racionalidad y la justicia de situaciones, de la propia práctica de la 
investigación acción participativa, se considera uno de los métodos de investigación- acción apuntada, como participativa (IAP), más utilizado en el presente de las acciones educativas, pues, es una investigación sumida en la problemática cotidiana, la cual implica técnicas y procedimientos de reflexión, transformación, aprendizaje y evaluación en la educación.(Teppa,2006).

En este orden de ideas, el estudio encuadra en un tipo de investigación acción participativa (IAP), porque la metodología permite desarrollar un análisis participativo, donde los actores comprometidos conjuntamente con las investigadoras se convierten en los protagonistas del proceso de construcción del conocimiento de la realidad sobre el objeto de estudio, en la detección de problemas y necesidades y en la elaboración de propuestas, soluciones y cambios. Busca lograr la participación de los actores sociales para que en la integración haya un proceso de desplazamiento actitudinal y reflexivo hacia conformación de brigadas de primeros auxilios para la enseñanza de las medidas preventivas.

Este estudio, se dirige según Teppa (op.cit), la cual, “ propone de acuerdo al uso de la investigación acción participativa (IAP) en el aula, la espiral autorreflexiva como sistema de ciclos sucesivos de" (p. 47): Este ciclo corresponde al momento 1 Diagnostico: revisar y estudiar documentos, Momento 2 Planificación: elaborar plan de acción, Momento 3 Observación-acción: Ejecución del plan y observaciones simultaneas, Momento 4 Reflexión: Valoración y evaluación del accionar continuo y Momento 5 Replanificación: Rectificación y reconstrucción del plan sistemáticamente

\section{Informantes Claves}

Martínez, (2004), asevera que "los informantes claves son los sujetos que se seleccionan para entrevistar, con la condición de que hayan vivido la situación estudiada y estén dispuestos a relatar esa experiencia desde el punto de vista cualitativo durante el proceso y dar cuenta de él, para así abordar el conocimiento desde una perspectiva natural.” (p. 56).En este estudio son tres (3) informantes claves (estudiantes del 5to grado "D”) 
Asimismo, la recolección de datos, es la toma de decisiones de cómo recoger información y se debe llegar a resultados generalizables, como no se puede generalizar la población completa se escoge una muestra de ella. El investigador establece distancia con los objetos de estudio. La investigación Acción participativa (IAP) está orientada a la solución de un problema definido por la comunidad, donde el investigador se compromete con el grupo. Al respecto, Tamayo y Tamayo (2009), la define como "la expresión operativa del diseño de investigación"(p. 126). Es importante señalar, que se ejecutó la evaluación diagnóstica (prueba escrita), para determinar los conocimientos previos que poseen los estudiantes sobre primeros auxilios.

Según Brenes (2006) citado por Torres (2010), la evaluación diagnóstica “es el conjunto de técnicas y procedimientos evaluativos que se aplican antes y durante el desarrollo del proceso de instrucción" (p.7). Esta técnica permite verificar los conocimientos e identificar las dificultades presentadas por cada uno de los estudiantes.

\section{Técnicas e Instrumentos}

De igual manera, se aplicó la encuesta en forma de cuestionario dirigida a los estudiantes (Cuadro 1), las mismas están conformadas por (4) cuatro ítems de respuestas abiertas, el cual Arias (ob. cit) define que una encuesta es "aquella donde el investigador puede obtener información acerca de un grupo de individuos" (p. 78), es decir, se puede tener información precisa. Cabe destacar, que el cuestionario de preguntas surgió de la categorización de los objetivos (ver cuadro 1), por su parte Alfonso (2012), señala que "la categorización es un proceso por medio del cual se busca reducir la información de la investigación con el fin de expresarla y describirla de manera conceptual” (p.3). Es decir, las categorías arrojan la información, el resultado o las necesidades educativas.

Alineado, el análisis de los datos recogidos en la presente investigación con la categorización la cual permitió etiquetar cada situación resaltante y frecuente que se presentó durante el proceso de recolección de datos. La categorización "consiste en resumir o sintetizar en una idea o concepto (una palabra o expresión breve pero elocuente) un 


\section{BRIGADA DE PRIMEROS AUXILIOS COMO ESTRATEGIA PARA LA ENSEÑANZA \\ DE MEDIDAS DE PREVENCIÓN DE LA SALUD ESCOLAR \\ Maritza Álvarez y Yolanda Montoya \\ (pp 57-82)}

conjunto de información escrita, grabada o filmada para su fácil manejo posterior" (Martínez, M., 2000)

\section{HALLAZGOS}

\section{Fase I. Diagnóstico}

Esta fase es donde se ubica el problema y se recoge la opinión del objeto de estudio, llamado también por Hurtado y Toro (1997) "diagnóstico participativo", porque es necesaria la intervención de los autores. En esta fase, se identificará el problema, se recolectan y procesan todas las informaciones relativas a él. En este trabajo de investigación, el diagnóstico se realizó desde el mes de noviembre, hasta el mes de octubre del siguiente año. La aplicó una entrevista semiestructurada a los estudiantes (3 informantes claves), donde las respuestas dadas evidenciaron la importancia e interés de conformar brigadas de primeros auxilios en su escuela ,resultado las categorías emergentes: Heridos , Aliviar Dolor , Atender Rápido , Accidente Medicamentos, Cruz Roja ,Proteger, Cuidar,, Peligros Formar equipos, Avisas los peligros ,Ayudar.

A continuación, se presenta el cuadro donde se registró el diagnóstico hecho a los estudiantes. 


\section{BRIGADA DE PRIMEROS AUXILIOS COMO ESTRATEGIA PARA LA ENSEÑANZA \\ DE MEDIDAS DE PREVENCIÓN DE LA SALUD ESCOLAR \\ Maritza Álvarez y Yolanda Montoya}

(pp 57-82)

\section{Cuadro 1}

Diagnóstico que evidencia la opinión de los estudiantes sobre la Brigada de Primeros Auxilios como medidas de prevención

\begin{tabular}{|c|c|c|c|c|}
\hline \multicolumn{5}{|c|}{$\begin{array}{l}\text { Categoría generadora de opiniones: Brigadas de Primeros Auxilios Básicos } \\
\text { ESTUDIANTES }\end{array}$} \\
\hline Preguntas claves & 1 & 2 & 3 & $\begin{array}{l}\text { Categorías } \\
\text { Emergentes }\end{array}$ \\
\hline $\begin{array}{l}\text { 1.- ¿Qué crees que } \\
\text { son } \\
\text { auxilios? }\end{array}$ & $\begin{array}{lr}\begin{array}{l}\text { Son las } \\
\text { que se }\end{array} & \text { hacen } \\
\text { cuando } & \text { hay } \\
\text { heridos } & \end{array}$ & $\begin{array}{lr}\text { Aliviar el } \\
\text { dolor de las } \\
\text { personas }\end{array}$ & $\begin{array}{l}\text { Atender } \\
\text { rápido en un } \\
\text { accidente }\end{array}$ & $\begin{array}{l}\text { Heridos } \\
\text { Aliviar Dolor } \\
\text { Atender Rápido } \\
\text { Accidente }\end{array}$ \\
\hline $\begin{array}{lr}2 .- & \text { Uds. } \\
\text { (estudiantes), ¿cuáles } \\
\text { acciones deben } \\
\text { tomarse r como } \\
\text { brigadista de } \\
\text { primeros auxilios? }\end{array}$ & $\begin{array}{l}\text { Pedir } \\
\text { colaboración } \\
\text { para los } \\
\text { medicamentos } \\
\text { de la Cruz Roja }\end{array}$ & $\begin{array}{l}\text { Cuidar y } \\
\text { proteger a los } \\
\text { que estudian } \\
\text { aquí }\end{array}$ & $\begin{array}{l}\text { Avisar los } \\
\text { peligros }\end{array}$ & $\begin{array}{l}\text { Medicamentos } \\
\text { Cruz Roja } \\
\text { Proteger } \\
\text { Cuidar } \\
\text { Peligros }\end{array}$ \\
\hline $\begin{array}{lr}\text { 3.- Sabes } & \text { cómo } \\
\text { actuar frente a una } \\
\text { emergencia } \\
\text { requiera que } \\
\text { Auxilios? }\end{array}$ & $\begin{array}{lr}\begin{array}{l}\text { Aprender } \\
\text { en la }\end{array} \\
\text { clase. } & \text { La } \\
\text { maestra } & \text { me } \\
\text { enseña } & \end{array}$ & $\begin{array}{l}\text { Tengo que } \\
\text { aprender a } \\
\text { los primeros } \\
\text { auxilios }\end{array}$ & $\begin{array}{l}\text { No sé mucho } \\
\text { pero, la } \\
\text { maestra me } \\
\text { enseñará } \\
\text { para hacerlo } \\
\text { bien }\end{array}$ & $\begin{array}{l}\text { Aprender } \\
\text { Primeros auxilios }\end{array}$ \\
\hline $\begin{array}{l}\text { 4.- ¿ Qué es una } \\
\text { brigada de primeros } \\
\text { auxilios? }\end{array}$ & $\begin{array}{lr}\text { Grupo de } & \text { niños } \\
\text { que } & \text { curan, } \\
\text { cuidarán } & \text { y } \\
\text { atienden } & \text { al } \\
\text { momento de } & \text { un } \\
\text { desmayo } & \end{array}$ & $\begin{array}{lr}\text { Es la que } & \text { se } \\
\text { encarga } & \text { de } \\
\text { avisar } & \text { los } \\
\text { peligro } & \end{array}$ & $\begin{array}{l}\text { Son personas } \\
\text { preparadas } \\
\text { para ayudar }\end{array}$ & $\begin{array}{l}\text { Formar equipos } \\
\text { Avisas los } \\
\text { peligros } \\
\text { Ayudar }\end{array}$ \\
\hline
\end{tabular}

Fuente: Álvarez y Montoya (2016)

\section{Fase II Planificación}

En este Momento 2, hay que organizar las acciones que se van a tomar en la investigación, cómo lo señala Teppa (op.cit.) "se elabora el plan, es decir, se inserta la etapa clásica de planificación pero se hace énfasis en el proceso de elaboración.”(p.6) Esta se origina a través de talleres con un plan a ejecutar en el aula de clases. En este momento también 
realizar la organización de las acciones ejecutar. Las cuales fueron actividad realizadas en el aula de clase para la formación, sensibilización conocimiento, concientizar y entrenar a los y las estudiantes para actuar de forma receptiva ante una situación de emergencia y preservar la salud tanto de ellos como de la personas de su entorno, generando un espíritu analítico reflexivo encaminado a potenciar la creatividad que conlleve a la enseñanza y aprendizaje significativo.

\section{Fase III. Ejecución de la Propuesta}

En el Momento 3, es donde se aplica lo planificado y se abordar la problemática para darle la solución de la manera directa. Como lo mencionan Hurtado y Toro (2007), es el "enfrentamiento con el o los problemas, es la que permite el conocimiento más profundo de la realidad, pues éste sólo se logra cuando se intenta transformarla” (p.149). La ejecución de las actividades programadas se llevó a cabo entre los meses de abril y junio de 2013.

De acuerdo a los objetivos planificados, se evidenció que se logró el propósito y cumplimiento de las actividades programadas donde se observó la integración y el interés por parte de los estudiantes para la debida asimilación de los temas tratados referente a la prevención de enfermedades, primeros auxilios, anatomía, salud, entre otras, donde se acreditó la ampliación de los conocimientos y el aprendizaje activo y significativo dentro del proceso enseñanza y aprendizaje.

Es evidente entonces, que la salud es uno de los denominados temas transversales que deben incluirse en todas las áreas del aprendizaje. Igualmente, importante es, que los y las estudiantes aprendan a tomar medidas en defensa de la salud individual y colectiva siendo una prioridad en la sociedad y por ende debe estar presente en los centros educativos.

En el marco de las consideraciones planteadas, las autoras concluyen que la implementación de la Brigada de Primeros Auxilios Básicos de Salud como estrategia innovadora facilita a los y las estudiantes del 5to grado " $D$ ”, la integración de la teoría con la práctica dando como resultado la obtención de un aprendizaje significativo Las actividades se ejecutaban en una semana se realizaba los martes a las ocho (8:00) de la mañana hasta las once y cincuenta y cinco (11:55); la semana siguiente se ejecutaba los 
días lunes, desdelas siete (7:00) de la mañana, hasta las once (11:00); para un total de cuatro (4) sesiones en un lapso 3 semanas.

Los objetivos propuestos fueron alcanzados satisfactoriamente, pues los estudiantes participaron de forma amena en las diferentes sesiones. Ejecutándose actividades teórico prácticas en el conocimiento de los aspectos relacionados con la toma de signos vitales, reconocimiento de signos y síntomas para la identificación de una enfermedad y las medidas preventivas para evitarlas. Además selogró que las clases y las actividades se desarrollaran de manera efectiva.

A continuación, se presenta el plan de acción ejecutado El momento 3, se trata de poner en práctica lo planificado y de abordar la problemática y la solución de misma de manera directa. Como lo mencionan Hurtado y Toro (1997): es el "enfrentamiento con el o los problemas, es la que permite el conocimiento más profundo de la realidad, pues éste sólo se logra cuando se intenta transformarla". La ejecución de las actividades programadas se llevó a cabo entre los meses de abril y junio de 2015.

En esta sección se da inicio a la sistematización de la Innovación Educativa, donde se describirán los resultados, seguimiento y evolución de las diferentes estrategias planificadas y desarrolladas para el aprendizaje y enseñanza de la promoción de la salud y la atención de primeros auxilios, con la finalidad de que los estudiantes de $5^{\circ}$ grado " $D$ " de la Escuela Básica Estadal "General Diego Ibarra”, participen de manera cooperativa y amena a través de la brigada escolar, para resolver situaciones de emergencia primaria de la salud que se presenten en el quehacer cotidiano escolar, donde se logre estimular el aprendizaje cognitivo que a su vez genere un aprendizaje significativo que potencie, desarrolle y fortalezca su pensamiento creativo

\section{Cuadro 2}

Plan de Estrategias para la Implementación de Brigadas Escolares de Primeros Auxilios a medidas preventivas 


\section{BRIGADA DE PRIMEROS AUXILIOS COMO ESTRATEGIA PARA LA ENSEÑANZA \\ DE MEDIDAS DE PREVENCIÓN DE LA SALUD ESCOLAR \\ Maritza Álvarez y Yolanda Montoya}

(pp 57-82)

Objetivo General. Implementar una brigada escolar de primeros auxilios básicos como herramienta para la enseñanza en salud de los y las estudiantes de $5^{\circ}$ grado "D" en la Escuela Básica Estadal “General Diego Ibarra”, Parroquia Miguel Peña Municipio Valencia Estado Carabobo.

\begin{tabular}{|c|c|c|c|c|}
\hline TIEMPO & OBJETIVO ESPECÍFICO & ESTRATEGIAS & RECURSOS & LOGRO \\
\hline 4 Horas & $\begin{array}{l}\text { Motivar al estudiante a } \\
\text { través de actividades teórico } \\
\text { prácticas en el conocimiento } \\
\text { de la labor que debe cumplir } \\
\text { el brigadista, su rol, } \\
\text { funciones y compromisos. }\end{array}$ & $\begin{array}{l}\text { Lectura reflexiva. } \\
\text { Discusión } \\
\text { socializada sobre el } \\
\text { rol del brigadista. } \\
\text { Dinámica de parada } \\
\text { como brigadista } \\
\text { escolar }\end{array}$ & $\begin{array}{ll}\text { Humanos: Docente, } \\
\text { estudiantes } \\
\text { Material: lápiz, } \\
\text { cuadernos, hojas, } \\
\text { láminas, } & \text { gorra, } \\
\text { distintivos } & \end{array}$ & $\begin{array}{l}\text { Involucrar a los } \\
\text { estudiantes del } \\
\text { 5to grado "D" en } \\
\text { la participación de } \\
\text { la brigada escolar } \\
\text { de primeros } \\
\text { auxilios básicos }\end{array}$ \\
\hline 4 Horas & $\begin{array}{l}\text { Desarrollar actividades } \\
\text { teórico prácticas en el } \\
\text { conocimiento de los } \\
\text { aspectos relacionados con la } \\
\text { toma de signos vitales, } \\
\text { reconocimiento de signos y } \\
\text { síntomas para la } \\
\text { identificación de una } \\
\text { enfermedad y las medidas } \\
\text { preventivas para evitarlas. }\end{array}$ & $\begin{array}{l}\text { Lectura reflexiva } \\
\text { Discusión } \\
\text { Socializada sobre } \\
\text { los pasos necesarios } \\
\text { para tomar la tensión } \\
\text { arterial, y signos } \\
\text { vitales Dinámica } \\
\text { interactiva } \\
\text { manipulación de } \\
\text { recursos médicos } \\
\text { para la identificación } \\
\text { primaria de una } \\
\text { enfermedad. } \\
\text { Sociodrama. }\end{array}$ & $\begin{array}{l}\text { Humanos } \\
\text { Docente, } \\
\text { estudiantes } \\
\text { Material: } \\
\text { lápiz, cuadernos, } \\
\text { hojas, láminas, } \\
\text { gorra, distintivos, } \\
\text { termómetro, } \\
\text { tensiómetro, } \\
\text { lecturas } \\
\text { relacionadas }\end{array}$ & $\begin{array}{l}\text { Capacitar a los } \\
\text { integrantes de la } \\
\text { brigadas escolar } \\
\text { con contenidos de } \\
\text { signos vitales y } \\
\text { tomar tensión } \\
\text { arterial }\end{array}$ \\
\hline 4 Horas & $\begin{array}{l}\text { Además se planificará para } \\
\text { el uso del botiquín de } \\
\text { primeros auxilios }\end{array}$ & $\begin{array}{l}\text { Lectura Reflexiva: } \\
\text { La Cooperación } \\
\text { Discusión } \\
\text { socializada Dinámica } \\
\text { de primeros auxilios } \\
\text { Simulacros en el aula } \\
\text { de clases }\end{array}$ & $\begin{array}{l}\text { Humanos: } \\
\text { Docente, } \\
\text { estudiantes } \\
\text { Material: } \\
\text { lápiz, cuadernos, } \\
\text { hojas, láminas, } \\
\text { gorra, distintivos } \\
\text { Implementos que } \\
\text { contiene el botiquín } \\
\text { de primeros } \\
\text { auxilios }\end{array}$ & $\begin{array}{l}\text { Comprometer a } \\
\text { los estudiantes y } \\
\text { docentes de la } \\
\text { E.B.E "General } \\
\text { Diego Ibarra", en } \\
\text { la brigada de } \\
\text { primeros auxilios } \\
\text { básicos para } \\
\text { servir a la } \\
\text { comunidad } \\
\text { escolar }\end{array}$ \\
\hline
\end{tabular}

Fuente: Álvarez, y Montoya (2016)

\section{Fase IV. Reflexiones (Análisis Final)}

En el momento 4, de transformación, se ajusta el plan según los resultados de la reflexión, se replanificaron actividades para las próximas clases de educación para la salud, sumándoles reflexiones sobre los valores preventivos y de cooperación, solidaridad y humanidad. Como resultado de las observaciones y reflexiones se modifican o transforman 


\section{BRIGADA DE PRIMEROS AUXILIOS COMO ESTRATEGIA PARA LA ENSEÑANZA \\ DE MEDIDAS DE PREVENCIÓN DE LA SALUD ESCOLAR \\ Maritza Álvarez y Yolanda Montoya}

ciertas estrategias, evaluaciones o recursos del plan. Se adicionó charlas por los estudiantes del 5to grado (objeto de estudio) a los 4to s y 6to grados sobre la importancia de brigada, salud, primeros auxilios, promoción de la salud y medidas preventivas a través del diálogo participativo donde los estudiantes expresan sus conocimientos previos y experiencias sobre el tema. También se les explica las funciones, roles, responsabilidades y compromisos de la brigada. Finalmente, se aplica una actividad donde los estudiantes en una hoja de ejercicios enumeran las situaciones o circunstancias en las que ellos creen que se deben prestar los primeros auxilios.

Talleres o actividades especiales como los círculos dialécticos para discutir los cambios, así como también se delibera con respecto a los resultados de la autoevaluación, coevaluación y reflexión (Teppa,2008).

\section{SISTEMATIZACIÓN DE LOS TALLERES}

Taller $N^{\circ} 1$ "La Importancia De Mis Signos Vitales

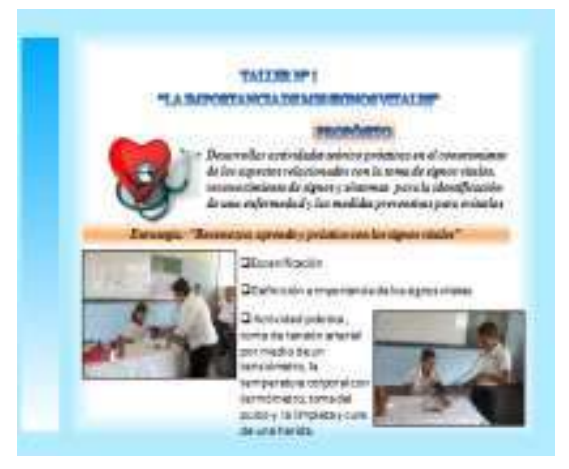

Resultado de la actividad y evaluación: La finalidad de la actividad realizada es estimular el conocimiento, sensibilizar, concientizar y entrenar a los y las estudiantes para actuar de forma receptiva ante una situación de emergencia y preservar la salud tanto de ellos como de la personas de su entorno, generando un espíritu analítico reflexivo encaminado a potenciar la creatividad que conlleve a la enseñanza y aprendizaje significativo 


\section{BRIGADA DE PRIMEROS AUXILIOS COMO ESTRATEGIA PARA LA ENSEÑANZA \\ DE MEDIDAS DE PREVENCIÓN DE LA SALUD ESCOLAR \\ Maritza Álvarez y Yolanda Montoya}

(pp 57-82)

\section{Taller N² “Conociendo Mi Cuerpo Aprendo A Cuidar Mi Salud”}

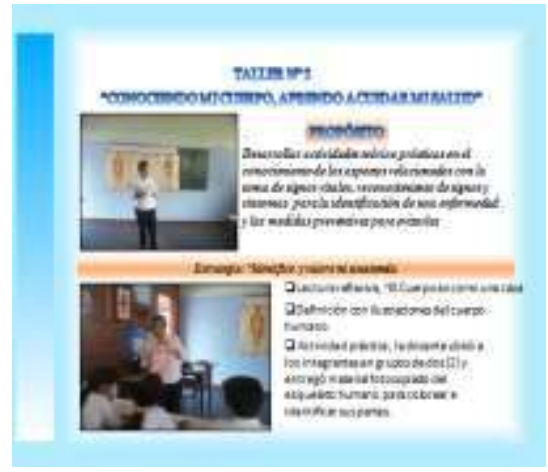

Resultados de la actividad y evaluación: La actividad se realizó con el objetivo didáctico de que los y las estudiantes aprendan a identificar las distintas partes del cuerpo humano, la importancia que conozca, explore $y$ vivencie, no sólo sus partes externas y visibles, sino también aquellas que no se ven, encaminándolos a un desarrollo integral por medio de actividades lúdicas y creativas bajo experiencias de cooperación y compañerismo

Taller $\mathbf{N}^{\circ} 3$ "Brigadistas Siempre Listos"

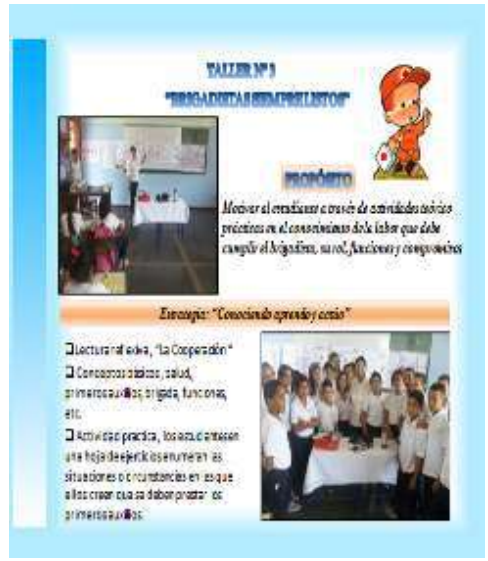

Resultados de la actividad y evaluación: Se observó gran motivación e interés de los y las estudiantes en torno los temas planteados además de la participación espontánea de los mismos, evidenciándose el conocimiento adquirido a través de la aplicación de la estrategia donde se busca consolidar el aprendizaje significativo. Rediseñar esta propuesta para todos los grados de Segunda Etapa de la escuela. 


\section{BRIGADA DE PRIMEROS AUXILIOS COMO ESTRATEGIA PARA LA ENSEÑANZA \\ DE MEDIDAS DE PREVENCIÓN DE LA SALUD ESCOLAR \\ Maritza Álvarez y Yolanda Montoya}

(pp 57-82)

\section{CONSIDERACIONES FINALES}

En esta investigación de la praxis educativa,es revelador el logro de la aplicabilidad del plan de acción en el aula, por transformar durante el ciclo de talleres, las enseñanzas pedagógicas, de aprendizaje significativos en los contenidos de educación para salud, referentes a los cuidados y autoconciencia sobre las propias necesidades de vivir situaciones de:heridos, accidentes, desastres, dolor, atención, además que se valoran las medidas preventivas.

Por su parte, la manera reflexiva de los estudiantes facilitó la formación en conocimientos de primeros auxilios mediante un proceso de argumentación teórico práctico, que se logró a través de las estrategias del plan de acción aplicadas en el aula, permitiendo transformar e innovar de manera constante, durante el ciclo de talleres, las enseñanzas pedagógicas, en aprendizajes significativos con los contenidos de educación para salud. Se pudo observar el interés de los estudiantes en aprender sobre el taller de primeros auxilios tanto en lo teórico como en lo práctico, además se observó el entusiasmo y sobre todo la colaboración prestada por dichos estudiante.

Como consecuencia, los estudiantes conformaron brigadas de primeros auxilios referidos a los cuidados y autoconciencia sobre las propias necesidades de vivirante situaciones de: heridos, accidentes, desastres, dolor, atención, lo cual, incentivo a distinguir entre lo que se debe y no debe hacerse, buscando condiciones de calidad de vida y protección de ella, apropiándose de las medidas preventivas, elementos importantes en el quehacer de las brigadas de primeros auxilios.

\section{REFERENCIAS}

Alfonso, N. (2012). Categorización. Documento en línea, disponible en: http://www.calameo.com/boocks/002784318d9be4e3f4d50.

Arias ,F .(2012) El Proyecto e Investigación. 8va edición. Editorial Episteme.

Volumen $22 N^{\circ} 1$ Enero-Abril 2018. ISSN: 2244-7296 


\section{BRIGADA DE PRIMEROS AUXILIOS COMO ESTRATEGIA PARA LA ENSEÑANZA \\ DE MEDIDAS DE PREVENCIÓN DE LA SALUD ESCOLAR \\ Maritza Álvarez y Yolanda Montoya}

(pp 57-82)

Ausubel, D. (1978). «In defense of advance organizers: A reply to the critics.» Review of Educational Research, 48, 251-257.

Carr, W. y Kemmis, S. (1983). Becoming critical. Knowing through action research. Deakin. Victoria, Australia: University Press

Carretero, M. (2001). Metacognición y Educación. Buenos Aires: Aique

Castro, R (2006). Primeros Auxilios. Texto en línea. Disponible: http://www.saludlatina.com/primeros-auxilios.html.Consulta: 20013, enero 20.

Constitución de la República Bolivariana de Venezuela.(1999) Publicada en Gaceta Oficial del jueves 30 de diciembre. Número 36.860

Cowman, S (1993). Triangulation. A Means of Reconciliation in Nursing Research. Journal of Advanced Nursing. Vol 18.

Cruz Roja Venezolana (2006). Día Mundial de Primeros Auxilios. Texto en línea. Disponible. http://www.cruzRojaVenezolana.com/Dia-mundial-de- PrimerosAuxilios.html.Consulta:2007.Febrero20.

Little, H. \& Wyver, S. (2010). Individual differences in children's risk perception and appraisals in outdoor play environments. International Journal of Early Years Education, 18, 297-313.

De Lima, Dinorah. "Nuevas ideas para viejas intenciones". México, 1992, p. 15. (Mecanograma.)

Estrada C y Otros (2011) "Brigada ecológica y de emergencia escolar en la unidad educativa Nuestra señora de Coromoto en la parroquia Naguanagua del estado Carabobo que permita garantizar la atención en niños, niñas y adolescentes y a la comunidad en general en caso de accidentes" Colegio Universitario de Los Teques "Cecilio Acosta", estado Miranda, Venezuela.

Fabris, L (s/f). Socorrista del SNS (ACNUR) y de la CRUZ ROJA ARGENTINA, Certificado en RCP avanzado de la Cruz Roja Argentina y Fundación Cardiológica Argentina. Buzo deportivo Cruz Roja CMAS. Colgate Palmolive Argentina.www.iztacala.unam.mx/www_fesi/proteccioncivil/Manual_Primeros_Auxilio s.[Consulta. Octubre 2013] 


\section{BRIGADA DE PRIMEROS AUXILIOS COMO ESTRATEGIA PARA LA ENSEÑANZA \\ DE MEDIDAS DE PREVENCIÓN DE LA SALUD ESCOLAR \\ Maritza Álvarez y Yolanda Montoya}

(pp 57-82)

Fundación Atención Inmediata (1999). Emergencias Médicas. Alfa Impresiones. Primera Edición Venezuela.http://www.monografias.com/trabajos-pdf5/campana-capacitacionprimeros-auxilios/campana-capacitacion-primeros-auxilios.shtml\#ixzz5BwkpT6xY

Gabari MI, Buitrago H. (2006) .Simulación y entrenamiento en hábitos de prevención de accidentes infantiles, demanda docente de recursos multimedia en Navarra (España). Norte de Santander, Colombia: Universidad de Pamplona

Hurtado, I. y Toro, J. (2007). Paradigmas y métodos de investigación en tiempos de cambio. Venezuela. Editorial CEC.

Informe del Comité de Expertos de la OMS sobre Educación y Promoción de la Salud Integral en la Escuela. Promover la salud en la escuela. Colección de Informes Técnicos nº 870, Ginebra, 1997

La Madriz, L y Jenniz La Madriz.(2013). Comunicación efectiva como estrategia didáctica para potenciar el proceso de enseñanza y aprendizaje. _Revista EDUCARE, Volumen 17, Número 1. Barquisimeto. pp.74-92

Ley de Educación Gaceta Oficial de la República Bolivariana de Venezuela Extraordinaria Numero 2.635 de fecha 26 de julio de 1.980, Ordinaria Número 36.787 de fecha 15 de septiembre de 1.999.

Ley Orgánica para la Protección del Nino, Niña y Adolescente, Gaceta Oficial Número 5.266 Extraordinario de Fecha de 02 de Octubre de 1.998

Manual de Emergencias Médicas Fundación Atención Inmediata (1995): Brigada de Salud Escolar. Programa Escuela Saludable. https://escuelasaludableguarico.wordpress.com/escuela-saludable/brigadas-de-saludescolar/

Marti, J. (2006). "La investigación-Acción Participativa. Estructura y fases" en Villasantes, T. (2000). "La investigación social participativa". Madrid: Ed. El viejo topomde2olk/paradigma-socio-critico/

Martínez, M., (2004). "La capacidad creadora y sus implicaciones para la metodología de la investigación”, Psicología (Caracas: UCV), vol.XII, núm.1-2, 37-62.

Morrongiello, B. \& Lasenby-Lessard, J. (2007). Psychological determinants of risk taking by children: an integrative model and implications for interventions. Injury prevention, $13,20-25$.

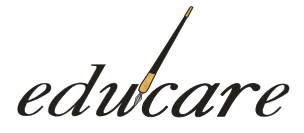

Volumen $22 \quad N^{\circ} 1$ Enero-Abril 2018. ISSN: 2244-7296 


\section{BRIGADA DE PRIMEROS AUXILIOS COMO ESTRATEGIA PARA LA ENSEÑANZA \\ DE MEDIDAS DE PREVENCIÓN DE LA SALUD ESCOLAR \\ Maritza Álvarez y Yolanda Montoya \\ (рp 57-82)}

Organización Mundial De La Salud (1978): Salud para todos. Declaración de Alma Ata. Ginebra: OMS.http://www.paho.org/spanish/dd/pin/alma-ata_declaracion.htm [Consulta:septiembre 2015].

Sotelo, K. (1 de 11 del 2012). prezi. Obtenido de prezi: http://prezi.com/_p7v

Tamayo, M. y Tamayo M. (2009) “Diccionario de la Investigación Científica”. Editorial Blanco, México.

Teppa, S. (2006). Investigación-acción participativa en la praxis pedagógica diaria.

Barquisimeto: Subdirección de Investigación y Postgrado de la UPEL-IPB. Excelencia Creativa

Teppa, S.(octubre de 2008). IV JORNADAS DE INVESTIGACIÓN E INNOVACIÓN EDUCATIVA “Creatividad e Innovación en Contextos Socioeducativos de Cambios". Universidad Centrooccidental Lisandro Alvarado (UCLA). Barquisimeto

Unión Internacional de promoción de la salud y educación para la salud UIPES. (2010). Promover la Salud en la Escuela: de la evidencia a la acción

Vygotsky L (1990) Caracterización del paradigma socioculturalhttp://es.slideshare.net/geraldineillingworth/teora-sociocultural-vygotsky

Vygotsky L (1991) El niño: consideraciones psicológicas y pedagógicas. Capítulo II. http://www.earlytechnicaleducation.org/spanien/cap2lis3es.htm 